\title{
TOPOLOGY OF THE CYCLIC ČECH-HOCHSCHILD BICOMPLEX
}

\author{
JAN KUBARSKI
}

\begin{abstract}
We define the cyclic Čech-Hochschild bicomplex for a good covering of a smooth manifold and calculate its homology using some nonstandard spectral sequences. The results show that its homology is very rich.
\end{abstract}

\section{Preface}

In classical differential topology and geometry, the double Čech-de Rham complex $\left(\left\{C^{p}\left(\mathfrak{U}, \Omega^{q}\right)\right\}_{p \geq 0, q \geq 0}, \delta, \tilde{d}\right)$ for a good covering $\mathfrak{U}$ of $M$ corresponds to the single de Rham complex $\left(\Omega^{*}(M), d\right)$ of $M$. The total cohomology of the double Cech-de Rham complex is isomorphic to the de Rham cohomology, therefore, the characteristic classes of $M$ could be determined by locally defined cochain forms from this Čech-de Rham bicomplex (see [1], [2, last two pages], [6]). Passing to noncommutative geometry on smooth manifolds (i.e. to noncommutative geometry of the algebra $C^{\infty}(M)$ of smooth functions on $\left.M\right)$, we see that the single Connes complex $\left(C_{*}^{\lambda}\left(C^{\infty}(M)\right), b^{\prime}\right)$ is the counterpart of the single de Rham complex. We can ask: which bicomplex corresponds to the single Connes complex in noncommutative geometry of smooth manifolds?

During his visit to the Institute of Mathematics of the Lodz University of Technology in December 2009 at the invitation of the author, N. Teleman suggested the definition (Def. 2.4 below) of the cyclic Čech-Hochschild bicomplex $\left(\left\{\check{C} H_{p, q}^{\lambda}(\mathfrak{U}, \mathbb{R})\right\}_{p \geq 0, q \geq 0}, \tilde{\delta}, b^{\prime}\right)$ that corresponds to the single Connes complex and to compute its total homology in connection with the problem of computing characteristic classes. This problem will be discussed elsewhere. In this paper we calculate the total homology of this bicomplex using some nonstandard spectral sequences. The results show that its homology is very rich, hence it contains the characteristic classes. The problem of finding suitable cyclic Čech-Hochschild chains representing the characteristic classes is still open.

$M S C$ (2010): primary 58B34, 16E40, 55U15, 57R20.

Keywords: double Cech-de Rham complex, good covering, noncommutative geometry on smooth manifolds, single Connes complex, cyclic complex, single de Rham complex, cyclic Čech-Hochschild bicomplex, Čech cohomology, cocycle, local formulas for characteristic classes, Hochschild homology, cyclic homology, Chern character. 


\section{Cyclic Čech-Hochschild Bicomplex, Definition}

The Connes complex $\left(C_{*}^{\lambda}\left(C^{\infty}(M)\right), b^{\prime}\right)$ of a smooth manifold consists of cyclic Hochschild chains over the algebra of smooth functions $C^{\infty}(M)$ and bar differentials

$$
\ldots \stackrel{b^{\prime}}{\longrightarrow} C_{q+1}^{\lambda}\left(C^{\infty}(M)\right) \stackrel{b^{\prime}}{\longrightarrow} C_{q}^{\lambda}\left(C^{\infty}(M)\right) \stackrel{b^{\prime}}{\longrightarrow} \ldots \stackrel{b^{\prime}}{\longrightarrow} C_{0}^{\lambda}\left(C^{\infty}(M)\right) .
$$

We recall that $C_{q}^{\lambda}\left(C^{\infty}(M)\right) \subset C_{q}\left(C^{\infty}(M)\right)=\left\{\phi^{q}: \prod^{q+1} M \rightarrow \mathbb{R} ; \phi^{q}\right.$ is smooth $\}$ and $b_{q}^{\prime}: C_{q}\left(C^{\infty}(M)\right) \rightarrow C_{q-1}\left(C^{\infty}(M)\right)$ is defined by the formula

$$
\left(b_{q}^{\prime}\left(\phi^{q}\right)\right)\left(x_{0}, \ldots, x_{q-1}\right)=\sum_{i=0}^{q-1}(-1)^{i} \phi^{q}\left(x_{0}, \ldots, x_{i}, x_{i}, \ldots, x_{q-1}\right)
$$

where $C_{q}^{\lambda}\left(C^{\infty}(M)\right)=\operatorname{ker}\left(1-T_{q}\right)$ is the subspace of cyclic $q$-chains, i.e., such that $T_{q}\left(\phi^{q}\right)=\phi^{q}$, where $T_{q}: C_{q}\left(C^{\infty}(M)\right) \rightarrow C_{q}\left(C^{\infty}(M)\right), T_{q}\left(\phi^{q}\right)\left(x_{0}, \ldots, x_{q}\right)=$ $(-1)^{q} \phi^{q}\left(x_{1}, \ldots, x_{q}, x_{0}\right)$ is the graded cyclic permutation operator.

The relationship between the above defined spaces of Hochschild $q$-chains and the abstract Hochschild theory of an arbitrary algebra $A$ consists in identifying $C_{q}\left(C^{\infty}(M)\right)$ with the tensor power $\widehat{\otimes}^{q+1} C^{\infty}(M)$ in the category of topological linear spaces (after introducing the Fréchet topology in $C^{\infty}(M)$ ), see [4]. The $q$ th homology group of the Connes complex is denoted by $\mathbf{H}_{q}^{\lambda}\left(C^{\infty}(M)\right)$. These homology groups were introduced by Connes [3] (for compact manifolds) and by Teleman [7] (for paracompact manifolds); see also [5].

Theorem 2.1 (Connes-Teleman theorem). For a paracompact manifold $M$, we have

$$
\mathbf{H}_{0}^{\lambda}\left(C^{\infty}(M)\right)=\Omega^{0}(M)=C^{\infty}(M),
$$

$$
\begin{aligned}
& \mathbf{H}_{2 i}^{\lambda}\left(C^{\infty}(M)\right) \quad \text { for } i \geq 1 \\
= & \mathbf{H}_{D R}^{0}(M) \oplus \mathbf{H}_{D R}^{2}(M) \oplus \mathbf{H}_{D R}^{4}(M) \oplus \ldots \oplus \mathbf{H}_{D R}^{2 i-2}(M) \oplus \Omega^{2 i}(M) / d\left[\Omega^{2 i-1}(M)\right], \\
& \mathbf{H}_{2 i+1}^{\lambda}\left(C^{\infty}(M)\right) \\
= & \mathbf{H}_{D R}^{1}(M) \oplus \mathbf{H}_{D R}^{3}(M) \oplus \mathbf{H}_{D R}^{5}(M) \oplus \ldots \oplus \mathbf{H}_{D R}^{2 i-1}(M) \oplus \Omega^{2 i+1}(M) / d\left[\Omega^{2 i}(M)\right] .
\end{aligned}
$$

Particularly, if $\operatorname{dim} M$ is even, $\operatorname{dim} M=2 m$, then

$$
\begin{aligned}
& \mathbf{H}_{2 m+2}^{\lambda}\left(C^{\infty}(M)\right)=\mathbf{H}_{2 m+4}^{\lambda}\left(C^{\infty}(M)\right)=\ldots \\
= & \mathbf{H}_{D R}^{0}(M) \oplus \mathbf{H}_{D R}^{2}(M) \oplus \mathbf{H}_{D R}^{4}(M) \oplus \ldots \oplus \mathbf{H}_{D R}^{2 m}(M),
\end{aligned}
$$

if $\operatorname{dim} M$ is odd, $\operatorname{dim} M=2 m+1$, then

$$
\begin{aligned}
& \mathbf{H}_{2 m+2}^{\lambda}\left(C^{\infty}(M)\right)=\mathbf{H}_{2 m+4}^{\lambda}\left(C^{\infty}(M)\right)=\ldots \\
= & \mathbf{H}_{D R}^{0}(M) \oplus \mathbf{H}_{D R}^{2}(M) \oplus \mathbf{H}_{D R}^{4}(M) \oplus \ldots \oplus \mathbf{H}_{D R}^{2 m}(M) .
\end{aligned}
$$

From this theorem we can deduce that the whole algebra of characteristic classes (and the Chern character) is sitting in one homology space, namely in $\mathbf{H}_{2 m+2}^{\lambda}\left(C^{\infty}(M)\right)$, where $m=\operatorname{dim} M$. For contractible sets $U \subset M$, we obtain in particular 
Conclusion 2.2 (Connes-Teleman theorem for contractible open sets). If $U \subset$ $M$ is a contractible open subset $\left(U \cong \mathbb{R}^{m}\right)$, then

$$
\mathbf{H}_{0}^{\lambda}\left(C^{\infty}(U)\right)=C^{\infty}(U),
$$

for $i \geq 1$

$\mathbf{H}_{2 i}^{\lambda}\left(C^{\infty}(U)\right)=\mathbb{R} \oplus\left(\Omega^{2 i}(U) / \operatorname{Im} d^{2 i-1}\right)=\mathbb{R} \oplus\left(\Omega^{2 i}(U) / \operatorname{ker} d^{2 i}\right)=\mathbb{R} \oplus \operatorname{Im} d^{2 i}$,

$\mathbf{H}_{2 i-1}^{\lambda}\left(C^{\infty}(U)\right)=\left(\Omega^{2 i-1}(U) / \operatorname{ker} d_{2 i-1}\right)=\operatorname{Im} d_{2 i-1}, \quad i \geq 1$.

Remark 2.3. (a) A constant function $C$ on $U$ transforms - via the above isomorphisms - to the cohomology class of the constant function $2^{i}(2 i+1)$ !! in the space $C_{2 i}^{\lambda}=\operatorname{ker}\left(1-T_{2 i}\right)$.

(b) Let $q=2 i$ or $2 i-1$. Take $U$ with a coordinate system $x=\left(x^{1}, \ldots, x^{m}\right)$. We can find a formula for the inclusion $\left(\Omega^{q}(U) / d^{q-1}\left[\Omega^{q-1}\right]\right) \longmapsto \mathbf{H}_{q}^{\lambda}\left(C^{\infty}(U)\right)$. For a $q$-differential form $\omega=\sum_{i_{1}, \ldots, i_{q}} \omega^{i_{1} \ldots i_{q}} d x^{i_{1}} \wedge \ldots \wedge d x^{i_{q}}$, we define a function $f_{\omega}=\sum_{i_{1} \ldots i_{q}} f_{i_{1} \ldots i_{q}}$ on $U^{q+1}$ where

$$
f_{i_{1} \ldots i_{q}}\left(x_{0}, x_{1}, \ldots, x_{q}\right)=\omega^{i_{1} \ldots i_{q}}\left(x_{0}\right) \frac{1}{q !} \sum_{\tau \in S_{q}} \operatorname{sgn} \tau \cdot\left(x_{\tau_{1}}^{i_{1}}-x_{0}^{i_{1}}\right) \cdot \ldots \cdot\left(x_{\tau_{q}}^{i_{q}}-x_{0}^{i_{q}}\right) .
$$

The class $\left[N_{q} f_{\omega}\right]$ in $\mathbf{H}_{q}^{\lambda}\left(C^{\infty}(U)\right)\left(N_{q}=\sum_{r=0}^{q}\left(T_{q}\right)^{r}\right)$ is the value of this inclusion on $[\omega]$. We observe that: 1$) b^{\prime}([C])=0$ and $\left.[C]=0 \Leftrightarrow C=0,2\right) b^{\prime}\left(\left[N_{q} f_{\omega}\right]\right)=0$ iff $\omega$ is exact, 3) if $V \subset U$ is also a subset such that $V \cong \mathbb{R}^{m}$, then $N_{q}\left(f_{\omega \mid V}\right)=$ $\left(N_{q} f_{\omega}\right) \mid V^{q+1}$, i.e., the restriction of a chain coming from a differential form comes from a differential form. The above three observations are the key to proving the collapsing of the spectral sequence of the $\lambda$-Čech-Hochschild bicomplex $\check{C} H^{\lambda}$ at the second term (see below).

In classical differential topology and geometry, the single de Rham complex of $M$ corresponds to the double Cech-de Rham complex for a good covering $\mathfrak{U}$ of $M$. And what about noncommutative geometry? N. Teleman proposes the following definition of the Čech-Hochschild bicomplex for a good covering $\mathfrak{U}$ of $M$ as follows:

Definition 2.4. By the (scalar) Čech-Hochschild bicomplex for a good covering $\mathfrak{U}$ of $M$ we mean the first quadrant bicomplex

$$
\check{C} H(\mathfrak{U}, \mathbb{R})=\left(\left\{\check{C} H_{p, q}(\mathfrak{U}, \mathbb{R})\right\}_{p \geq 0, q \geq 0}, \tilde{\delta}, b^{\prime}\right)
$$

where $\check{C} H_{p, q}(\mathfrak{U}, \mathbb{R})=\bigoplus_{\left(i_{0}, \ldots, i_{p}\right) \in I^{p+1}}\left\{\phi_{i_{0} \ldots i_{p}}^{q}: \prod^{q+1} U_{i_{0} \ldots i_{p}} \rightarrow \mathbb{R}\right\}$ and the differentials $b^{\prime}, \tilde{\delta}$ are defined as follows:

a) $b_{p, q}^{\prime}$, the bar differential $(p \geq 0, q \geq 1)$ of bidegree $\left.(0,-1)\right)$ :

$$
\begin{gathered}
b_{p, q}^{\prime}: \check{C} H_{p, q}(\mathfrak{U}, \mathbb{R}) \rightarrow \check{C} H_{p, q-1}(\mathfrak{U}, \mathbb{R}), \\
\left(b_{p, q}^{\prime}\left(\phi_{p}^{q}\right)\right)_{i_{0}, \ldots, i_{p}}\left(x_{0}, \ldots, x_{q-1}\right)=\sum_{i=0}^{q-1}(-1)^{i} \phi_{i_{0} \ldots i_{p}}^{q}\left(x_{0}, \ldots, x_{i}, x_{i}, \ldots, x_{q-1}\right) .
\end{gathered}
$$

b) $\delta_{p, q}$, the Čech differential $(p, q \geq 0)$ of bidegree $(+1,0)$ :

$$
\begin{gathered}
\delta_{p, q}: \check{C} H_{p, q}(\mathfrak{U}, \mathbb{R}) \rightarrow \check{C} H_{p+1, q}(\mathfrak{U}, \mathbb{R}), \\
\delta_{p, q}\left(\phi^{q}\right)_{i_{0}, \ldots, i_{p+1}}=\sum_{r=0}^{p+1}(-1)^{r} \phi_{i_{0}, \ldots, \hat{i_{r}}, \ldots, i_{p+1}}^{q} \mid U_{i_{0}, \ldots, i_{p+1}} .
\end{gathered}
$$


It is a standard calculation that $\delta b^{\prime}=b^{\prime} \delta$. By setting $\tilde{\delta}_{p, q}=(-1)^{q} \delta_{p, q}$, we introduce the so-called $\check{C}$ ech-Hochschild bicomplex $\left(\check{C} H(\mathfrak{U}, \mathbb{R}), \tilde{\delta}, b^{\prime}\right)$.

We take the graded cyclic permutation $T_{p, q}: \check{C} H_{p, q}(\mathfrak{U}, \mathbb{R}) \rightarrow \check{C} H_{p, q}(\mathfrak{U}, \mathbb{R})$, $T_{p, q}\left(\phi^{q}\right)_{i_{0}, \ldots, i_{p}}\left(x_{0}, \ldots, x_{q}\right)=(-1)^{q} \phi_{i_{0} \ldots i_{p}}^{q}\left(x_{1}, \ldots, x_{q}, x_{0}\right)$ and cyclic functions

$$
\check{C} H_{p, q}^{\lambda}(\mathfrak{U}, \mathbb{R})=\left\{\phi \in \check{C} H_{p, q}(\mathfrak{U}, \mathbb{R}) ; T_{p, q}(\phi)=\phi\right\}=\operatorname{ker}\left(1-T_{p, q}\right) .
$$

The spaces of cyclic chains are stable under the bar and Čech differentials, and we obtain the cyclic Čech-Hochschild bicomplex

$$
\begin{aligned}
& \check{C} H^{\lambda}(\mathfrak{U}, \mathbb{R})=\left(\left\{\check{C} H_{p, q}^{\lambda}(\mathfrak{U}, \mathbb{R})\right\}_{p, q}, \tilde{\delta}, b^{\prime}\right)
\end{aligned}
$$

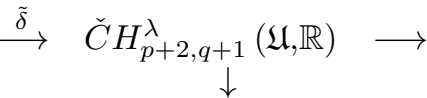

$$
\begin{aligned}
& \stackrel{\tilde{\delta}}{\longrightarrow} \quad \check{C} H_{p, q}^{\lambda}(\mathfrak{U}, \mathbb{R}) \quad \stackrel{\tilde{\delta}}{\longrightarrow} \quad \check{C} H_{p+1, q}^{\lambda}(\mathfrak{U}, \mathbb{R}) \quad \longrightarrow
\end{aligned}
$$

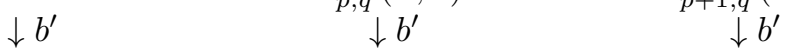

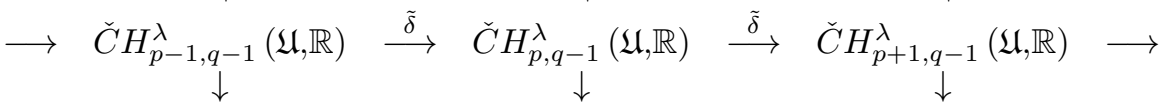

We observe that each $p$-column is the direct sum of the Connes complexes for the contractible sets $U_{i_{0} \ldots i_{p}}=U_{i_{0}} \cap \ldots \cap U_{i_{p}}$

$$
\begin{gathered}
\downarrow b^{\prime} \\
C_{q+1}^{\lambda}\left(C^{\infty}\left(U_{i_{0} \ldots i_{p}}\right)\right) \\
\downarrow b^{\prime} \\
C_{q}^{\lambda}\left(C^{\infty}\left(U_{i_{0} \ldots i_{p}}\right)\right) \\
\downarrow b^{\prime} \\
C_{q-1}^{\lambda}\left(C^{\infty}\left(U_{i_{0} \ldots i_{p}}\right)\right) \\
\downarrow b^{\prime}
\end{gathered}
$$

Therefore the above mentioned Connes-Teleman theorem on homology of the complex $C_{*}^{\lambda}\left(C^{\infty}\left(U_{i_{0} \ldots i_{p}}\right)\right)$ is very useful in the homological research of this complex. We observe that the total differential $D=\tilde{\delta}+b^{\prime}$ is homogeneous of degree +1 with respect to the total gradation along the lines $p-q=$ const, see Figure 1 , (in

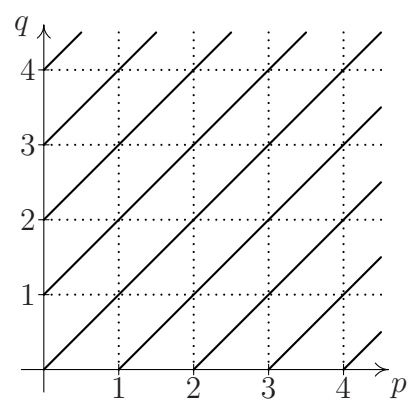

Figure 1. Total gradation. 
contrast to the Čech-de Rham complex where the total differential is homogeneous of degree +1 with respect to the total gradation along $p+q=$ const). These lines contain infinitely many spaces (unlike the Čech-de Rham complex). We introduce the total gradation by Cartesian product (not by direct sum)

$$
C^{[r]}=\prod_{p-q=r} \check{C} H_{p, q}^{\lambda}(\mathfrak{U}, \mathbb{R}), D^{[r]}: C^{[r]} \rightarrow C^{[r+1]}, \quad D^{2}=0, r \in \mathbb{Z} .
$$

Our main problem is:

Problem 2.5. Calculate the total homology of the above (scalar) Cech-Hochschild bicomplex (to show the possibility of finding the characteristic classes by the homology of this bicomplex, i.e., the possibility of finding Čech-Hochschild chains representing characteristic classes).

Using some nonstandard spectral sequences introduced below, we are able to calculate these total homologies.

\section{H-Bicomplex - AN Algebraic point of VIEW on ČECH-Hochschild BICOMPLEXES}

Definition 3.1. By an $H$-bicomplex $\mathcal{C}=\left(\left\{C_{p, q}\right\}_{p \geq 0, q \geq 0}, \tilde{\delta}, b^{\prime}\right)$, we mean a matrix $\left\{C_{p, q}\right\}_{p \geq 0, q \geq 0}$ of vector spaces together with two collections $\tilde{\delta}, b^{\prime}$ of mappings such that $\tilde{\delta}_{p, q}: C_{p, q} \rightarrow C_{p+1, q}$ and $b_{p, q}^{\prime}: C_{p, q} \rightarrow C_{p, q-1}, \tilde{\delta}, b^{\prime}$ are differential operators $\tilde{\delta}^{2}=0,\left(b^{\prime}\right)^{2}=0$, and $\tilde{\delta} b^{\prime}+b^{\prime} \tilde{\delta}=0$.

The Čech-Hochschild bicomplex $\check{C} H(\mathfrak{U}, \mathbb{R})$ and the cyclic Čech-Hochschild bicomplex $\check{C} H^{\lambda}(\mathfrak{U}, \mathbb{R})$ are examples of $H$-bicomplexes. The total differential

$$
D=\tilde{\delta}+b^{\prime}: \bar{C}^{[r]} \rightarrow \bar{C}^{[r+1]}, r \geq 0,
$$

is homogeneous of degree +1 with respect to the total gradation by Cartesian product along the lines $p-q=$ const,

$$
\bar{C}^{[r]}:=\prod_{p=q=r} C_{p, q} \subset \prod_{p, q} C_{p, q}=: \bar{C} ; D^{2}=0 .
$$

The total homology of $\left(\bar{C}^{[\bullet]}, D\right)$ will be denoted by $\mathbf{H}^{[r]}(\bar{C}):=\operatorname{ker} D^{[r]} / \operatorname{Im} D^{[r-1]}$, and we have

$$
\mathbf{H}(\bar{C}, D)=\prod_{r} \mathbf{H}^{[r]}(\bar{C}) .
$$

Assume the following notation: an infinite sequence $\left(x_{p}\right)_{p \geq 0}$ (an element of the Cartesian product) will be denoted as an infinite sum $\sum x_{p}$. Below we explain why we must consider Cartesian products (not direct sums) in the definition of total gradation and, consequently we must consider Cartesian products in the global description of the $H$-bicomplex: $\bar{C}:=\prod_{p, q} C_{p, q}$. To this end, consider the $q$ th row

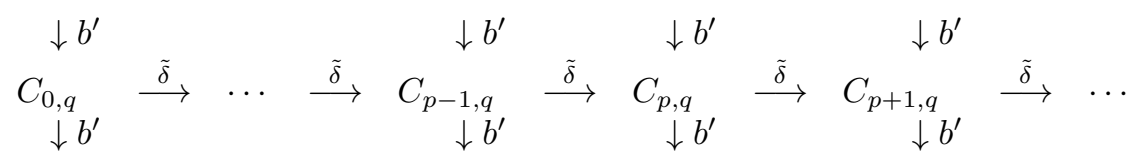


and the one induced in $b^{\prime}$-homology,

$$
\mathbf{H}_{q}\left(C_{0, *}, b^{\prime}\right) \stackrel{\tilde{\delta}_{\#}}{\rightarrow} \mathbf{H}_{q}\left(C_{1, *}, b^{\prime}\right) \stackrel{\tilde{\delta}_{\#}}{\rightarrow} \ldots \stackrel{\tilde{\delta}_{\#}}{\rightarrow} \mathbf{H}_{q}\left(C_{p, *}, b^{\prime}\right) \stackrel{\tilde{\delta}_{\#}}{\rightarrow} \mathbf{H}_{q}\left(C_{p+1, *}, b^{\prime}\right) \stackrel{\tilde{\delta}_{\#}}{\rightarrow} \ldots
$$

Theorem 3.2 (Theorem on total triviality). If all above rows (3.1) are exact, then each $D$-cycle $x=\sum_{k=0}^{\infty} x_{i+k, j+k}$ for $i \geq 1, j \geq 0, D(x)=0$ (of total degree $r=i-j)$ is D-exact, i.e., there exists an element $y \in C^{[r-1]}$ such that $D y=x$. (REMARK: The sequence $y=\sum_{k=0}^{\infty} y_{i-1+k, j+k}$ is infinite even if the sequence $x=\sum_{k=0}^{\infty} x_{i+k, j+k}$ is finite - this is why we need to consider the description via Cartesian products).

Proof. It is sufficient to consider the case $i=j$, i.e., $r=0$. Let $x$ be a $D$-cycle, so we have the equalities $b^{\prime}\left(x_{1,1}\right) \stackrel{(1)}{=} 0, \tilde{\delta}\left(x_{1,1}\right)+b^{\prime}\left(x_{2,2}\right) \stackrel{(2)}{=} 0, \tilde{\delta}\left(x_{2,2}\right)+b^{\prime}\left(x_{3,3}\right) \stackrel{(3)}{=}$ $0, \tilde{\delta}\left(x_{3,3}\right)+b^{\prime}\left(x_{4,4}\right) \stackrel{\left(3^{\prime}\right)}{=} 0$, etc. By $(2), \tilde{\delta}\left(x_{1,1}\right)=-b^{\prime}\left(x_{2,2}\right)$, so $\tilde{\delta}_{\#}\left(\left[x_{1,1}\right]\right)=0$. By the exactness of $(3.1),\left[x_{1,1}\right]=\tilde{\delta}_{\#}\left(\left[x_{0,1}\right]\right)$ for some $x_{0,1} \in C_{0,1}$ such that $\tilde{\delta}\left(x_{0,1}\right)=0$. Therefore $\left[x_{1,1}-\tilde{\delta}\left(x_{0,1}\right)\right]=0$. Then, there exists $x_{1,2} \in C_{1,2}$ such that $x_{1,1}-\tilde{\delta}\left(x_{0,1}\right) \stackrel{(4)}{=} b^{\prime}\left(x_{1,2}\right)$. This means that $x_{1,1}=\tilde{\delta}\left(x_{0,1}\right)+b^{\prime}\left(x_{1,2}\right)$. Consider now $b^{\prime} \delta_{2}\left(x_{1,2}\right)=-\tilde{\delta} b^{\prime}\left(x_{1,2}\right) \stackrel{(4)}{=}-\tilde{\delta}\left(x_{1,1}-\tilde{\delta}\left(x_{0,1}\right)\right)=-\tilde{\delta}\left(x_{1,1}\right) \stackrel{(2)}{=} b^{\prime}\left(x_{2,2}\right)$. Then, $b^{\prime}\left(x_{2,2}-\tilde{\delta}\left(x_{1,2}\right)\right)=0$ and $\tilde{\delta}\left(x_{2,2}-\tilde{\delta}\left(x_{1,2}\right)\right)=\tilde{\delta}\left(x_{2,2}\right) \stackrel{(3)}{=}-b^{\prime}\left(x_{3,3}\right)=b^{\prime}\left(-x_{3,3}\right)$. This yields $\tilde{\delta}_{\#}\left(\left[x_{2,2}-\delta_{2}\left(x_{1,2}\right)\right]\right)=0$. By exactness of rows, there exists $z_{1,2} \in C_{1,2}$ such that $b^{\prime}\left(z_{1,2}\right)=0$ and $\tilde{\delta}_{\#}\left(\left[z_{1,2}\right]\right)=\left[\tilde{\delta}\left(z_{1,2}\right)\right]=\left[x_{2,2}-\tilde{\delta}\left(x_{1,2}\right)\right]$. This means that $\left[\tilde{\delta}\left(z_{1,2}\right)-x_{2,2}+\tilde{\delta}\left(x_{1,2}\right)\right]=0$, so there exists $x_{2,3}$ such that $\tilde{\delta}\left(z_{1,2}\right)-x_{2,2}+$ $\tilde{\delta}\left(x_{1,2}\right)=b^{\prime}\left(-x_{2,3}\right)$. So, $x_{2,2} \stackrel{(5)}{=} \tilde{\delta}\left(x_{1,2}+z_{1,2}\right)+b^{\prime}\left(x_{2,3}\right)$, and $x^{1,1}=\tilde{\delta}\left(x_{0,1}\right)+$ $b^{\prime}\left(x_{1,2}+z_{1,2}\right)$. Consider now $b^{\prime} \tilde{\delta}\left(x_{2,3}\right)=-\tilde{\delta} b^{\prime}\left(x_{2,3}\right) \stackrel{(5)}{=}-\tilde{\delta}\left(x_{2,2}-\tilde{\delta}\left(x_{1,2}+z_{1,2}\right)\right)$ $=-\tilde{\delta}\left(x_{2,2}\right) \stackrel{(3)}{=} b^{\prime}\left(x_{3,3}\right)$. Then, $\quad b^{\prime}\left(x_{3,3}-\delta_{2}\left(x_{2,3}\right)\right)=0, \delta_{2}\left(x_{3,3}-\delta_{2}\left(x_{2,3}\right)\right)=$ $\delta_{2}\left(x_{3,3}\right) \stackrel{\left(3^{\prime}\right)}{=}-b^{\prime}\left(x_{4,4}\right)=b^{\prime}\left(-x_{4,4}\right)$. This yields $\tilde{\delta}_{\#}\left(\left[x_{3,3}-\tilde{\delta}\left(x_{2,3}\right)\right]\right)=0$. By exactness of rows, there exists $z_{2,3} \in C_{2,3}$ such that $b^{\prime} z_{2,3}=0$ and $\tilde{\delta}_{\#}\left(\left[z_{2,3}\right]\right)=$ $\left[\tilde{\delta}\left(z_{2,3}\right)\right]=\left[x_{3,3}-\tilde{\delta}\left(z_{2,3}\right)\right]$. This means that $\left[\tilde{\delta}\left(z_{2,3}\right)-x_{3,3}+\tilde{\delta}\left(x_{2,3}\right)\right]=0$, so there exists $x_{3,4}$ such that $\tilde{\delta}\left(z_{2,3}\right)-x_{3,3}+\tilde{\delta}\left(x_{2,3}\right)=b^{\prime}\left(-x_{3,4}\right)$. So, $x_{3,3}=$ $\tilde{\delta}\left(z_{2,3}+x_{2,3}\right)+b^{\prime}\left(x_{3,4}\right)$, and $x_{2,2}=\tilde{\delta}\left(x_{1,2}+z_{1,2}\right)+b^{\prime}\left(x_{2,3}+z_{2,3}\right)$, and so on. In this way, we obtain $y=x_{0,1}+\sum_{k=1}\left(x_{k, k+1}+z_{k, k+1}\right) \in \prod C_{k, k+1}$ such that $D(y)=x$.

Let $\mathcal{C}=\left(\left\{C_{p, q}\right\}_{p \geq 0, q \geq 0}, \tilde{\delta}, b^{\prime}\right)$ be an $H$-bicomplex. We introduce the Cartesian products $\bar{C}=\prod_{p, q=0}^{\infty} A_{p, q}, \bar{C}^{p}=\prod_{q=0}^{\infty} C_{p, q}$. We see that $\bar{C}=\prod_{p=0}^{\infty} \bar{C}^{p}$ and we say that $\bar{C}=\prod_{p=0}^{\infty} \bar{C}^{p}$ is a Cartesian-graded space. We introduce a decreasing filtration $\bar{C}_{j}, \quad j \geq 0$, by the Cartesian gradation $\bar{C}_{j}=\prod_{p \geq j}^{\infty} \bar{C}^{p} \subset \bar{C}, \bar{C}=\bar{C}_{0} \supset$ $\bar{C}_{1} \supset \ldots$ We have $\bar{C}_{j} / \bar{C}_{j+1}=\bar{C}^{j}$. Let us consider the induced filtered differential space $\left(\bar{C}, D, \bar{C}_{j}\right)$ (where $\left.D=\tilde{\delta}+b^{\prime}\right)$ and its spectral sequence $\left(\bar{E}_{s}^{j}, d_{s}\right)$. We recall 
some standard definitions: $\bar{Z}_{s}^{j}=\bar{A}_{j} \cap D^{-1}\left[\bar{A}_{j+s}\right], \bar{D}_{s}^{j}=\bar{A}_{j} \cap D\left[\bar{A}_{j-s}\right], \bar{E}_{s}^{j}=$ $\bar{Z}_{s}^{j} /\left(\bar{Z}_{s-1}^{j+1}+\bar{D}_{s-1}^{j}\right)$. Then, $D\left[\bar{Z}_{s}^{j}\right] \subset \bar{Z}_{s}^{j+s}$. Let $\eta: \bar{Z}_{s}^{j} \rightarrow \bar{E}_{s}^{j}$ be the projection. For each $s \geq 0$, there exists a linear homomorphism $d_{s}^{j}: \bar{E}_{s}^{j} \rightarrow \bar{E}_{s}^{j+s}$ such that $\eta \circ \delta=d_{s}^{j} \circ \eta: \bar{Z}_{s}^{j} \rightarrow \bar{E}_{s}^{j+s}$. Standard calculations give

Theorem 3.3. The first terms of the above spectral sequence are equal to:

(1) $\bar{E}_{0}^{j} \cong \bar{C}^{j}, d_{0}=b^{\prime}$, therefore, $\bar{E}_{0} \cong \bar{C}$ where $\bar{E}_{0}=\prod_{j=0}^{\infty} \bar{E}_{0}^{j}$ and $\bar{C}=\prod_{j=0}^{\infty} \bar{C}^{j}$

(2) $\bar{E}_{1}^{j} \cong \mathbf{H}^{j}\left(\bar{C}, b^{\prime}\right), d_{1}=\tilde{\delta}_{\#} ;$

(3) $\bar{E}_{2}^{j}=\mathbf{H}^{j}\left(\mathbf{H}\left(\bar{C}, b^{\prime}\right), \tilde{\delta}_{\#}\right)$.

One can see that we need to modify the total space of terms of the spectral sequence $\bar{E}_{s}$ by using products instead of direct sums, $\bar{E}_{s}:=\prod_{j=0}^{\infty} \bar{E}_{s}^{j}$. Now we use the total gradation along the lines $p-q=$ const, $\bar{C}^{[r]}=\prod_{p-q=r} C_{p, q}, \bar{C}_{j}^{[r]}=$ $\bar{C}_{j} \cap \bar{C}^{[r]}=\prod_{p \geq j, q=r}^{p-q \geq 0} \underset{p, q}{ } C_{\text {, where }} \bar{C}_{j}=\prod_{r \in \mathbb{Z}} \bar{C}_{j}^{[r]}$. Therefore,

$$
\bar{C}_{j}^{[r]}=\left\{\begin{array}{c}
\bar{C}_{j+1}^{[r]} \oplus C_{j, j-r} \text { for } j \geq r \\
\bar{C}_{j+1}^{[r]} \text { for } j<r
\end{array},\right.
$$

so, for all $i \in \mathbb{Z}$,

$$
\bar{E}_{0}^{j, i}=\bar{C}_{j}^{[j+i]} / \bar{C}_{j+1}^{[j+i]}=\left\{\begin{array}{c}
C_{j,-i}, i \leq 0 \\
\varnothing, \quad i>0 .
\end{array},\right.
$$

in other words $\bar{E}_{0}^{j, r-j}=\bar{C}_{j}^{[r]}(\bar{C}) / \bar{C}_{j+1}^{[r]}(\bar{C})$ (the total gradation in $\bar{E}_{0}$ is of course standardly defined, $\left.\bar{E}_{0}^{(r)}=\prod_{j+i=r} \bar{E}_{0}^{j, i}\right)$.

For the filtered differential space $\left(\bar{C}, D, \bar{C}_{j}\right)$, we introduce in the total homology $\mathbf{H}(\bar{C}, D)$ :

- the Cartesian gradation $\mathbf{H}(\bar{C}, D)=\prod_{r \in \mathbb{Z}} \mathbf{H}^{[r]}(\bar{C})$ and

- the usual filtration $\mathbf{H}_{j}(\bar{C})=\left\{[z] ; z \in \bar{C}_{j}\right\} \subset \mathbf{H}(\bar{C}, D)$, obtaining the socalled $\mathbb{Z}$-Cartesian graded filtered space. Standard calculations give the $\infty$-term $\bar{E}_{\infty}^{j}$ of the spectral sequence $\bar{E}_{\infty}^{j} \cong E_{0}^{j}(\mathbf{H}(\bar{C})):=\mathbf{H}_{j}(\bar{C}) / \mathbf{H}_{j+1}(\bar{C})$, therefore,

$$
\bar{E}_{\infty}=\prod_{j \geq 0} \bar{E}_{\infty}^{j} \cong \prod_{j \geq 0} E_{0}^{j}(\mathbf{H}(C))=\bar{E}_{0}(\mathbf{H}(\bar{C})) .
$$

Remark 3.4. In the classical theory of graded filtered modules $\left(A, A^{r}, A_{j}\right)$ we have the assumption $A_{j}^{r}=0$ for $r<j$. Therefore, the total gradation of $E_{0}^{(r)}(A)$ is finite, $E_{0}^{(r)}(A)=\bigoplus_{j=0}^{r} E_{0}^{j, r-j}$, and hence there exists a noncanonical linear isomorphism $A^{r} \equiv E_{0}^{(r)}(A)$. Applying this to the graded filtered homology modules $\left(\mathbf{H}(A)=\bigoplus \mathbf{H}^{r}(A), \mathbf{H}_{j}(A)\right)$, we obtain $\mathbf{H}^{r}(A) \stackrel{\equiv}{\rightarrow} E_{0}^{(r)}(\mathbf{H}(A))=$ $\bigoplus_{j=0}^{r} E_{0}^{j, r-j}(\mathbf{H}(A))$ and so there is a noncanonical linear isomorphism $\mathbf{H}(A) \stackrel{\equiv}{\rightarrow}$ 
$E_{0}(\mathbf{H}(A))$ homogeneous with respect to the gradation in $\mathbf{H}(A)$ and the total gradation in $E_{0}(\mathbf{H}(A))$.

In our theory of $H$-bicomplexes for the Cartesian-graded filtered homology space $\left(\mathbf{H}(\bar{C}, D)=\prod_{r \in \mathbb{Z}} \mathbf{H}^{[r]}(\bar{C}), \mathbf{H}_{j}(\bar{C})\right)$, the equality $\mathbf{H}_{j}^{[r]}(\bar{C})=0$ cannot occur (see Figure 2).

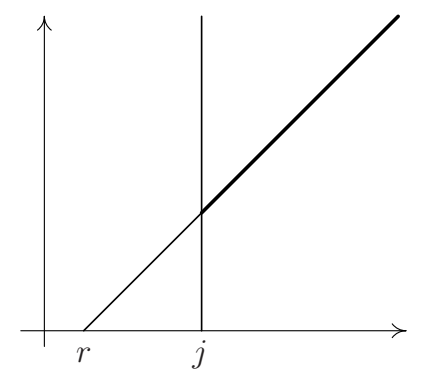

Figure 2. The equality $\mathbf{H}_{j}^{[r]}(\bar{C})=0$ cannot occur.

Therefore, we cannot claim that $\mathbf{H}^{[r]}(\bar{C}) \stackrel{\equiv}{\rightarrow} \bar{E}_{0}^{(r)}(\mathbf{H}(C)):=\prod_{j \geq 0} E_{0}^{j, r-j}(\mathbf{H}(C))$. However, we shall show below that in the particular example of the cyclic CechHochschild bicomplex the above condition does hold.

Definition 3.5. We say that a $H$-bicomplex $\mathcal{A}=\left(\left\{A_{p, q}\right\}_{p \geq 0, q \geq 0}, \tilde{\delta}, b^{\prime}\right)$ is $h o$ mologically regular if, for each $r \in \mathbb{Z}$, there exists $j \geq 0$ such that $\mathbf{H}_{j}^{[r]}(\bar{A})=0$.

Theorem 3.6. If the H-bicomplex $\mathcal{C}=\left(\left\{C_{p, q}\right\}_{p \geq 0, q \geq 0}, \tilde{\delta}, b^{\prime}\right)$ is homologically regular, then $E_{0}^{(r)}(\mathbf{H}(\bar{C}))=\prod_{j \geq 0} E_{0}^{j, r-j}(\mathbf{H}(\bar{C}))$ has a finite gradation and there exists an isomorphism $\psi: \mathbf{H}(\overline{\bar{C}}) \rightarrow \bar{E}_{0}(\mathbf{H}(\bar{C}))$ homogeneous with respect to the gradation in $\mathbf{H}(\bar{C})$ and the total gradation in $\bar{E}_{0}(\mathbf{H}(\bar{C}))$.

Now we give a condition on an $H$-bicomplex that

- guarantees homological regularity,

- is fulfilled for the cyclic Čech-Hochschild bicomplex $\check{C} H^{\lambda}(\mathfrak{U}, \mathbb{R})$.

Definition 3.7. We say that an $H$-bicomplex $\mathcal{C}=\left(\left\{C_{p, q}\right\}_{p \geq 0, q \geq 0}, \tilde{\delta}, b^{\prime}\right)$ has a regular zone if there exists a natural number $N$ such that, for all $q \geq N$, the sequences

$$
\mathbf{H}_{q}\left(C_{N, *}, b^{\prime}\right) \stackrel{\tilde{\delta}_{\#}}{\rightarrow} \mathbf{H}_{q}\left(C_{N+1, *}, b^{\prime}\right) \stackrel{\tilde{\delta}_{\#}}{\rightarrow} \ldots \stackrel{\tilde{\delta}_{\#}}{\rightarrow} \mathbf{H}_{q}\left(C_{p, *}, b^{\prime}\right) \stackrel{\tilde{\delta}_{\#}}{\rightarrow} \mathbf{H}_{q}\left(C_{p+1, *}, b^{\prime}\right) \stackrel{\tilde{\delta}_{\#}}{\rightarrow} \ldots
$$

are exact. By a regular $N$-zone, we then mean the submatrix $\left\{C_{p, q}\right\}$ for $p \geq N$, $q \geq N$. 
Remark 3.8. The existence of a regular $N$-zone implies that the submatrix $\mathcal{C}^{\prime}:=\left(\left\{C_{p, q}\right\}_{p \geq N, q \geq N}, \tilde{\delta}, b^{\prime}\right)$ satisfies the assumptions of Theorem 3.2 on total triviality.

If an $H$-bicomplex $\mathcal{A}=\left(\left\{C_{p, q}\right\}_{p \geq 0, q \geq 0}, \tilde{\delta}, b^{\prime}\right)$ has a regular $N$-zone for some integer $N$, then, for every integer $r$, there exists $j$ such that $\bar{C}_{j}^{[r]}$ is contained in the regular $N$-zone (i.e. in the submatrix $\left\{C_{p, q}\right\}_{p \geq N, q \geq N}$ ). Therefore, we obtain the following conclusion.

Theorem 3.9. If an $H$-bicomplex $\mathcal{C}=\left(\left\{C_{p, q}\right\}_{p \geq 0, q \geq 0}, \tilde{\delta}, b^{\prime}\right)$ has a regular $N$ zone, then, for each $r \in \mathbb{N}$, there exists $j$ such that $\mathbf{H}_{j}^{[r]}(\bar{C})=0$, i.e. $\mathcal{C}$ is homologically regular. Consequently, $\bar{E}_{0}(\mathbf{H}(\bar{C}))=\mathbf{H}(\bar{C})$.

\section{Topology of the Cyclic Čech-Hochschild BiCOMplex}

Let us return to the cyclic Čech-Hochschild bicomplex $\check{C} H^{\lambda}(\mathfrak{U}, \mathbb{R})$ and $b^{\prime}$-homology of its rows

$\mathbf{H}_{q}\left(\check{C} H_{0, *}^{\lambda}, b^{\prime}\right) \stackrel{\tilde{\delta}_{\#}}{\rightarrow} \mathbf{H}_{q}\left(\check{C} H_{1, *}^{\lambda}, b^{\prime}\right) \stackrel{\tilde{\delta}_{\#}}{\rightarrow} \ldots \stackrel{\tilde{\delta}_{\#}}{\rightarrow} \mathbf{H}_{q}\left(\check{C} H_{p, *}^{\lambda}, b^{\prime}\right) \stackrel{\tilde{\delta}_{\#}}{\rightarrow} \mathbf{H}_{q}\left(\check{C} H_{p+1, *}^{\lambda}, b^{\prime}\right) \stackrel{\tilde{\delta}_{\#}}{\rightarrow} \ldots$

where $\mathbf{H}_{q}\left(\check{C} H_{p, *}^{\lambda}(\mathfrak{U}, \mathbb{R}), b^{\prime}\right)=\bigoplus_{\alpha_{0} \ldots \alpha_{p}} \mathbf{H}_{q}^{\lambda}\left(C^{\infty}\left(U_{\alpha_{0} \ldots \alpha_{p}}\right)\right)$. Since $U_{\alpha_{0} \ldots \alpha_{p}}$ is contractible, by the Connes-Teleman theorem 2.2 we have

Theorem 4.1. The cyclic Čech-Hochschild bicomplex $\left(\check{C} H_{p, q}^{\lambda}(\mathfrak{U}, \mathbb{R}), \tilde{\delta}, b^{\prime}\right)$ has a regular $N$-zone with $N=\operatorname{dim} M+1$.

Proof. Indeed, in dimension $n \geq N$, there are no differential forms (except zero), therefore, the above rows (starting from number $N$ )

$$
\begin{array}{r}
\mathbf{H}_{q}\left(\check{C} H_{N, *}^{\lambda}, b^{\prime}\right) \stackrel{\tilde{\delta}_{\#}}{\rightarrow} \mathbf{H}_{q}\left(\check{C} H_{N+1, *}^{\lambda}, b^{\prime}\right) \stackrel{\tilde{\delta}_{\#}}{\rightarrow} \ldots \\
\stackrel{\tilde{\delta}_{\#}}{\rightarrow} \mathbf{H}_{q}\left(\check{C} H_{p, *}^{\lambda}, b^{\prime}\right) \stackrel{\tilde{\delta}_{\#}}{\rightarrow} \mathbf{H}_{q}\left(\check{C} H_{p+1, *}^{\lambda}, b^{\prime}\right) \stackrel{\tilde{\delta}_{\#}}{\rightarrow} \ldots
\end{array}
$$

are alternately zero (for odd $q$ ) and consist only of Čech cocycles (for even $q$ ) in dimensions greater than the dimension of the manifold, therefore, all the rows are exact.

Let us return to the spectral sequence of the cyclic Cech-Hochschild bicomplex $\check{C} H^{\lambda}(\mathfrak{U}, \mathbb{R})$. By the above notations, we have

$$
\begin{aligned}
\bar{E}_{0}^{j, i} & =\left\{\begin{array}{c}
\left(\check{C} H^{\lambda}\right)_{j, .-i} \text { for } i \leq 0 \\
0 \quad \text { for } i>0
\end{array}\right. \\
\bar{E}_{1}^{j, i} & =\left\{\begin{array}{c}
\mathbf{H}^{-i}\left(\check{C} H_{j, *}^{\lambda}, b^{\prime}\right) \text { for } i \leq 0 \\
0 \quad \text { for } i>0,
\end{array}\right. \\
\bar{E}_{2}^{j, i} & =\left\{\begin{array}{c}
\mathbf{H}^{j}\left(\mathbf{H}^{-i}\left(\check{C} H_{j, *}^{\lambda}, b^{\prime}\right), \tilde{\delta}_{\#}\right) \\
0 \text { for } i>0 .
\end{array}\right.
\end{aligned}
$$


The total gradation in the spectral-sequence terms $\bar{E}_{s}^{j, i}$ is defined along the lines $p+q=$ const,$\quad \bar{E}_{s}^{(r)}=\prod_{p+q=r} \bar{E}_{s}^{j, i}$. By the Connes-Teleman theorem 2.2, we have the first term of the spectral sequence $\bar{E}_{s}^{j, i}$ :

Theorem 4.2 (see Figure 3).

$$
\begin{aligned}
\bar{E}_{1}^{j, 2 i} & = \begin{cases}\check{C}(\mathfrak{U}) \bigoplus \bigoplus_{\alpha_{0} \ldots \alpha_{j}} \Omega^{-2 i}\left(U_{\alpha}\right) / \operatorname{ker} d^{-2 i}, & \text { for } i \leq 0, \\
0 & \text { for } i>0,\end{cases} \\
\bar{E}_{1}^{j, 2 i-1} & = \begin{cases}\bigoplus_{\alpha_{0} \ldots \alpha_{j}} \Omega^{-2 i+1}\left(U_{\alpha}\right) / \operatorname{ker} d^{-2 i+1}, & \text { for } i \leq 0, \\
0 & \text { for } i>0 .\end{cases}
\end{aligned}
$$

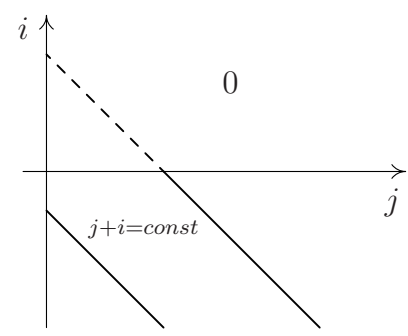

Figure 3. Table $\bar{E}^{1}$.

Let $\Omega_{j, q}=\bigoplus_{\alpha_{0} \ldots \alpha_{j}} \Omega^{q}\left(U_{\alpha_{0} \ldots \alpha_{j}}\right)$, consider the complex induced by the Čech differential

$$
\begin{aligned}
& \Omega_{0, q} / \operatorname{ker} d^{q} \stackrel{\delta}{\rightarrow} \Omega_{1, q} / \operatorname{ker} d^{q} \stackrel{\delta}{\rightarrow} \ldots \stackrel{\delta}{\rightarrow} \Omega_{j-1, q} / \operatorname{ker} d^{q} \\
& \stackrel{\delta}{\rightarrow} \Omega_{j, q} / \operatorname{ker} d^{q} \stackrel{\delta}{\rightarrow} \Omega_{j+1, q} / \operatorname{ker} d^{q} \stackrel{\delta}{\rightarrow} \ldots
\end{aligned}
$$

and let $\tilde{\mathbf{H}}^{j}\left(\Omega_{*, q} / \operatorname{ker} d^{q}, \delta_{\#}\right)$ be its cohomology denoted for brevity by $\tilde{\mathbf{H}}^{j, q}(M)$.

Theorem 4.3 (Andrzej Czarnecki, student of Robert Wolak, Jagiellonian University, private communication). The cohomology spaces $\tilde{\mathbf{H}}^{j, q}(M)$ are equal to

(a) $\tilde{\mathbf{H}}^{0, q}=\tilde{\mathbf{H}}^{0}\left(\Omega_{*, q} / \operatorname{ker} d, \delta_{\#}\right)=\mathbf{H}^{0}\left(Z^{*, q+1}, \delta\right)=\operatorname{ker} \delta^{0, q+1}=\operatorname{ker} d^{q+1}=$ $Z^{q+1}(M)$, in particular, $\tilde{\mathbf{H}}^{0, q}=0$ if $q+1 \geq \operatorname{dim} M$;

(b) for $j \geq 1, \tilde{\mathbf{H}}^{j, q}=\tilde{\mathbf{H}}^{j}\left(\Omega_{*, q} / \operatorname{ker} d, \delta_{\#}\right)=\mathbf{H}^{j}\left(Z^{*, q+1}, \delta\right)=\mathbf{H}^{j+q+1}(M)$, in particular $\tilde{\mathbf{H}}^{j, q}=0$ for $j+q+1 \geq \operatorname{dim} M+1$, i.e. for $j+q \geq \operatorname{dim} M$ (for example if $q \geq \operatorname{dim} M$ ).

Proof. Denote the de Rham differential $d: \Omega_{j, q} \rightarrow \Omega_{j, q+1}$ by $d^{j, q}$. We notice that (for $B^{j, q+1}=\bigoplus_{\alpha_{0} \ldots \alpha_{j}} d^{q}\left[\Omega^{q}\left(U_{\alpha_{0} \ldots \alpha_{j}}\right)\right], Z^{j, q+1}=\bigoplus_{\alpha_{0} \ldots \alpha_{j}} \operatorname{ker} d_{\alpha_{0} \ldots \alpha_{j}}^{q+1}$ )

$$
\Omega_{j, q} / \operatorname{ker} d^{j, q} \stackrel{\cong}{ } \operatorname{Im} d^{j, q}=B^{j, q+1}=Z^{j, q+1}=\operatorname{ker} d^{j, q+1}
$$


(since each $U_{\alpha_{0} \ldots \alpha_{j}}$ is contractible), therefore, we have the commutative diagram

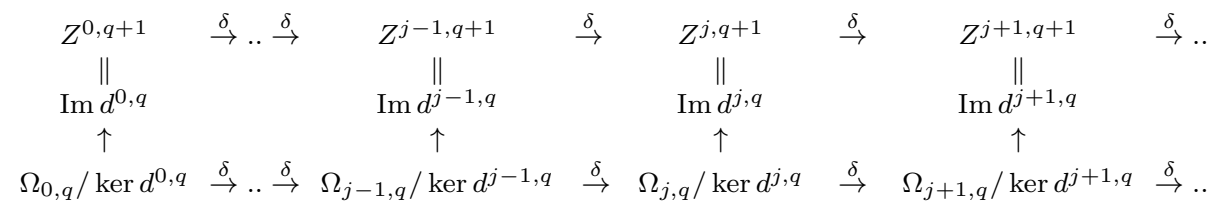

Therefore,

$$
\tilde{\mathbf{H}}^{j, q}=\tilde{\mathbf{H}}^{j}\left(\Omega_{*, q} / \operatorname{ker} d, \delta_{\#}\right)=\mathbf{H}^{j}\left(Z^{*, q+1}, \delta\right) .
$$

Now we can consider the truncated de Rham bicomplex:

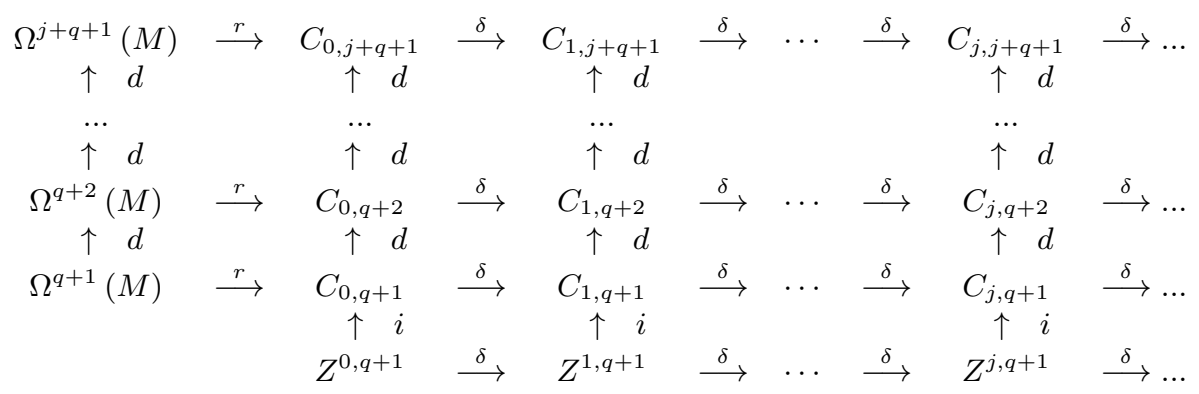

As the exactness of rows and columns is preserved, we know (from the MayerVietoris principle) that the above truncated de Rham bicomplex computes both the homology of the first column and of the first row. Thus, we get (a) and (b).

As a conclusion, we have the following equalities for the second term $\bar{E}_{2}^{j, i}$ :

Conclusion 4.4. Even rows.

For $j=0$

$$
\bar{E}_{2}^{0,2 i}=\left\{\begin{array}{ccc}
\check{\mathbf{H}}^{0}(M) \bigoplus_{Z^{-2 i+1}(M)} & \text { for } & 0 \leq-2 i \leq \operatorname{dim} M \\
\check{\mathbf{H}}^{0}(M) & \text { for } & -2 i>\operatorname{dim} M \\
0 & \text { for } & i>0,
\end{array}\right.
$$

i.e. $\quad \bar{E}_{2}^{0,-2 i}=\check{\mathbf{H}}^{0}(M) \bigoplus Z^{2 i+1}(M) \quad$ for $\quad i \geq 0$.

For $1 \leq j \leq \operatorname{dim} M$

$$
\bar{E}_{2}^{j, 2 i}=\left\{\begin{array}{ccc}
\check{\mathbf{H}}^{j}(M) \underset{\bigoplus^{j-2 i+1}(M)}{\breve{H}^{j-2 i}} & \text { for } & 0 \leq-2 i \leq \operatorname{dim} M \\
\check{\mathbf{H}}^{j}(M) & \text { for } & -2 i>\operatorname{dim} M \\
0 & \text { for } & i>0,
\end{array}\right.
$$

i.e. $\quad \bar{E}_{2}^{j,-2 i}=\check{\mathbf{H}}^{j}(M) \bigoplus \mathbf{H}^{j+2 i+1}(M) \quad$ for $\quad i \geq 0$.

Odd rows. For $j=0$

$$
\bar{E}_{2}^{0,2 i-1}=\left\{\begin{array}{c}
Z^{-2 i+2}(M) \quad \text { for } \quad i \leq 0, \quad 0 \leq-2 i+1 \leq \operatorname{dim} M \\
0 \quad \text { for } \quad i>0, \quad \text { or }-2 i+1>\operatorname{dim} M,
\end{array}\right.
$$

i.e. $\quad \bar{E}_{2}^{0,-2 i-1}=Z^{2 i+2}(M)$ for $i \geq 0$. 
For $1 \leq j \leq \operatorname{dim} M$

$$
\begin{aligned}
\bar{E}_{2}^{j, 2 i-1} & =\left\{\begin{array}{c}
\mathbf{H}^{j-2 i+2}(M) \text { for } i \leq 0, \quad 0 \leq-2 i+1 \leq \operatorname{dim} M \\
0 \quad \text { for } \quad i>0, \quad \text { or }-2 i+1>\operatorname{dim} M,
\end{array}\right. \\
\text { i.e. } \quad & \bar{E}_{2}^{j,-2 i-1}=\mathbf{H}^{j+2 i+2}(M) \text { for } i \geq 0,
\end{aligned}
$$

and, for $j>\operatorname{dim} M$, we have

$$
\begin{aligned}
& \bar{E}_{2}^{j, i}=\left\{\begin{array}{cc}
\mathbf{H}^{j-i+1}(M) & \text { for } 0 \leq-i \leq \operatorname{dim} M \\
0 & \text { otherwise, }
\end{array}\right. \\
& \text { i.e. } \quad \bar{E}_{2}^{j,-i}=\mathbf{H}^{j+i+1}(M) \quad \text { for } i \geq 0 .
\end{aligned}
$$

For example, if $N=2 m=\operatorname{dim} M=\left(\right.$ even), then the bottom half $\bar{E}_{2}^{j, i \leq 0}$ of $\bar{E}_{2}$ is as follows

$\begin{array}{cccccccc}i \backslash j & 0 & 1 & \cdots & N-1 & N & N+1 & \cdots \\ 0 & \check{H}^{0} \oplus \tilde{H}^{0,0} & \check{H}^{1} \oplus \tilde{H}^{1,0} & \cdots & \check{H}^{N-1} \oplus \tilde{H}^{N-1,0} & \check{H}^{N} \oplus \tilde{H}^{N, 0} & \tilde{H}^{N+1,0} & \ldots \\ -1 & \tilde{H}^{0,1} & \tilde{H}^{1,1} & \cdots & \tilde{H}^{N-1,1} & \tilde{H}^{N, 1} & \tilde{H}^{N+1,1} & \cdots \\ & \vdots & \vdots & & \vdots & \vdots & \vdots & \\ -(N-1) & \tilde{H}^{0, N-1} & \tilde{H}^{1, N-1} & \ldots & \tilde{H}^{N-1, N-1} & \tilde{H}^{N, N-1} & \tilde{H}^{N+1, N-1} & \cdots \\ -N & \check{H}^{0} \oplus \tilde{H}^{0, N} & \check{H}^{1} \oplus \tilde{H}^{1, N} & \cdots & \check{H}^{N-1} \oplus \tilde{H}^{N-1, N} & \check{H}^{N} \oplus \tilde{H}^{N, N} & \tilde{H}^{N+1, N} & \cdots \\ -(N+1) & 0 & 0 & & 0 & 0 & 0 & \cdots \\ -(N+2) & \check{H}^{0} & \check{H}^{1} & \ldots & \check{H}^{N-1} & \check{H}^{N} & 0 & \cdots \\ -(N+3) & 0 & 0 & & 0 & 0 & 0 & \cdots\end{array}$

Theorem 4.5. The spectral sequence of the cyclic Čech-Hochschild H-bicomplex $\check{C} H^{\lambda}(\mathfrak{U}, \mathbb{R})$ collapses at the second term, i.e. $d_{2}=d_{3}=\ldots=0$.

Proof. $\mathbf{H}^{q}\left(C_{p, *}^{\lambda}, b^{\prime}\right)=\bigoplus_{i_{0} \ldots i_{p}} \mathbf{H}^{q}\left(C_{i_{0} \ldots i_{p}, *}^{\lambda}, b^{\prime}\right), \mathbf{H}^{q}\left(C_{i_{0} \ldots i_{p}, *}^{\lambda}, b^{\prime}\right)$ is represented by (a) constant functions (if $q$ is $2 j$ ) or (b) by $N(f \omega)$ for a $q$-differential form $\omega \in \Omega^{q}\left(U_{i_{0} \ldots i_{p}}\right)$ (more exactly by $\left.[\omega]_{\operatorname{ker} d_{R}} \in \Omega^{q}\left(U_{i_{0} \ldots i_{p}}\right) / \operatorname{ker} d_{R}\right)$. The differential $d_{2}$ is linear, therefore, we can consider cases (a) and (b) independently. (a): Consider a constant function $C \in C_{i_{0} \ldots i_{p}, q}^{\lambda}(q=2 i)$. Then, the Čech differential $\delta(C)$ is the sum (sequence) of constant functions in $C_{\alpha i_{0} \ldots i_{p}, q}$ (on $U_{\alpha i_{0} \ldots i_{p}}^{q+1}$ ), $C_{i_{0} \alpha i_{1} \ldots i_{p}}, \ldots C_{i_{0} \ldots i_{j} \alpha i_{j+1} \ldots i_{p}}$, and in $C_{i_{0} \ldots i_{p} \alpha}$. Assuming that $[C] \in \mathbf{H}^{q}\left(C_{i_{0} \ldots i_{p}, *}^{\lambda}, b^{\prime}\right)$ is such that $\delta^{\#}([C])=0$, we find that $\delta(C)_{i_{0} \ldots \alpha \ldots i_{p}}$ - in each space $C_{i_{0} \ldots i_{j} \alpha i_{j+1} \ldots i_{p}}$ - is constant, therefore, $\delta(C)_{i_{0} \ldots \alpha \ldots i_{p}}=0$ (see Remark $\left.2.3 \mathrm{~b} 1\right)$ ). To define $d_{2}$, we should take an arbitrary $f \in C_{i_{0} \ldots \alpha \ldots i_{p}, q+1}^{\lambda}$ such that $b^{\prime}(f)=-\delta(C)_{i_{0} \ldots \alpha \ldots i_{p}}$, therefore, we can take $f=0$. Since the value of $d_{2}([C])$ can be taken as the class of $\delta(f)=0$, it follows that $d_{2}([C])=0$. (b): Consider a chain $\phi^{q}$ coming from a differential form $\omega \in \Omega^{q}\left(U_{i_{0} \ldots i_{p}}\right)$. Then (see Remark 2.3 b3) ) the Cech differential $\delta\left(\phi^{q}\right)$ is the sum (sequence) of chains coming from the restriction of the differential form $\omega$. Assuming that $\delta^{\#}([\omega])=0$, we deduce (see Remark 2.3 b2)) that $\delta\left(\phi^{q}\right)_{i_{0} \ldots \alpha \ldots i_{p}}$ comes from an exact differential form. Considering $\alpha \in\left\{i_{0}, \ldots, i_{p}\right\}$ we see that we can take the same exact differential form for all $\alpha$. Thus, $\left[\phi^{q}\right]=0$ and $d_{2}=0$. All the more, the equality $d_{3}=0$ holds etc., $d_{3}=d_{4}=\ldots=0$. 
The above two theorems yield:

Conclusion 4.6. For the cyclic $\check{C}$ ech-Hochschild bicomplex $\check{C} H^{\lambda}(\mathfrak{U}, \mathbb{R})$ we have $\bar{E}_{2}=\bar{E}_{3}=\ldots=\bar{E}_{\infty}=\bar{E}_{0}(\mathbf{H}(A))=\mathbf{H}(A)$. So $\bar{E}_{2}=\mathbf{H}(A), \quad \bar{E}_{2}^{(r)}=\mathbf{H}^{[r]}(A)$. Using the above calculations of $E_{2}^{(r)}$, we see that $\mathbf{H}^{[r]}(A)$ contains all the Čech cohomology groups. Then of course, $\mathbf{H}^{\text {even }}\left(\check{C} H^{\lambda}\right)$ and $\mathbf{H}^{\text {odd }}\left(\check{C} H^{\lambda}\right)$ contain the Čech cohomology $\bigoplus_{p} \check{H}^{2 p}$ and $\bigoplus_{p} \check{H}^{2 p+1}$ respectively.

Conclusion 4.7. Using the calculations of the second term $\bar{E}_{2}^{j, i}$ of the spectral sequence considered for the cyclic $\check{C}$ ech-Hochschild bicomplex $\check{C} H^{\lambda}(\mathfrak{U}, \mathbb{R})$, we have calculated its total homology. For example, if $\operatorname{dim} M=2 m$ then

$$
\begin{gathered}
\tilde{\mathbf{H}}^{0, q}(M)=\operatorname{ker} d^{q+1}=Z^{q+1}(M) \\
\tilde{\mathbf{H}}^{j, q}(M)=\mathbf{H}^{j+q+1}(M) \quad \text { for } j \geq 1 \\
\mathbf{H}^{[0]}(\bar{A})=\bar{E}_{2}^{(0)}=\bigoplus_{p=0}^{m} \check{\mathbf{H}}^{2 p}(M) \oplus \bigoplus_{p=0}^{2 m} \tilde{\mathbf{H}}^{p, p}(M) \\
=\bigoplus_{p=0}^{m} \check{\mathbf{H}}^{2 p}(M) \oplus\left(Z^{1}(M) \oplus \bigoplus_{p=1}^{m-1} \mathbf{H}^{2 p+1}\right) \\
\mathbf{H}^{[1]}(\bar{A})=\bar{E}_{2}^{(1)}=\bigoplus_{p=0}^{m-1} \check{\mathbf{H}}^{2 p+1}(M) \oplus \bigoplus_{p=0}^{2 m} \tilde{\mathbf{H}}^{p+1, p}(M) \\
=\bigoplus_{p=0}^{m-1} \check{\mathbf{H}}^{2 p+1}(M) \oplus \bigoplus_{p=0}^{m-1} \mathbf{H}^{2 p+2}(M) \\
\mathbf{H}^{[-1]}(\bar{A})=\bar{E}_{2}^{(-1)}=\bigoplus_{p=0}^{m-1} \check{\mathbf{H}}^{2 p+1}(M) \oplus \bigoplus_{p=0}^{2 m} \tilde{\mathbf{H}}^{p-1, p}(M) \\
=\bigoplus_{p=0}^{m-1} \check{\mathbf{H}}^{2 p+1}(M) \oplus \bigoplus_{p=0}^{m} \mathbf{H}^{2 p}(M) \\
E T C .
\end{gathered}
$$

Conclusion 4.8. The total homology of the cyclic Čech-Hochschild bicomplex $\check{C} H^{\lambda}(\mathfrak{U}, \mathbb{R})$ is very rich and, hence, contains the characteristic classes.

Remark 4.9. Considering from the beginning a $G$-cocycle $\left\{g_{\alpha \beta}\right\} g_{\alpha \beta}: U_{\alpha \beta} \rightarrow$ $G$, we produce nonscalar cochains. For example, if $G=G L(n) \subset \mathcal{M}_{n}(\mathbb{R})$, we can define cochains in the algebra of square matrices $\mathcal{M}_{n}(\mathbb{R}), C_{p, q}\left(\mathfrak{U}, \mathcal{M}_{n}(\mathbb{R})\right)=$ $\bigoplus_{\left(i_{0}, \ldots, i_{p}\right) \in I^{p+1}}\left\{\phi_{i_{0} \ldots i_{p}}^{q}:\left(U_{i_{0} \ldots i_{p}}\right)^{q+1} \rightarrow \mathcal{M}_{n}(\mathbb{R})\right\}$. The operators $T, \delta, b^{\prime}$ can be introduced by the same formulae. Considering any base of the vector space $\mathcal{M}_{n}(k)$, we see that these operators act on the coordinates, therefore, all the above theory of spectral sequences and total homology can be repeated and we have $\mathbf{H}^{[r]}\left(\bar{C} ; \mathcal{M}_{n}(\mathbb{R})\right)=\mathbf{H}^{[r]}(\bar{C}) \otimes \mathcal{M}_{n}(\mathbb{R})$. 
Remark 4.10. With each $\mathcal{M}_{n}(\mathbb{R})$-cochain $\phi$, we can associate a scalar cochain using the trace operator $\operatorname{Tr} \circ \phi$. The trace operator is invariant with respect to the adjoint representation, therefore, it plays a fundamental role for characteristic classes (among them, for the Chern character). One also notices that the cochain $\operatorname{Tr} \circ \phi$ can be a $D$-cycle [ $D$ being the total differential $]$ even if $\phi$ is not a cycle. Such a situation occurs for a variety of $G$-cochains specified by the cocycle $\left\{g_{\alpha \beta}\right\}$ (since $\operatorname{Tr}(A B)=\operatorname{Tr}(B A)$ but, in general, $A B \neq B A$ for $A, B \in G=G L(n))$.

\section{REFERENCES}

[1] R. Bott, On the Chern-Weil homomorphism and the continuous cohomology of Lie groups, Adv. Math. 11 (1973), 289-303.

[2] R. Bott and L. Tu, Differential Forms in Algebraic Topology, GTM, Springer-Verlag, 1982.

[3] A. Connes, Non-commutative differential geometry, Publ. Math. I.H.E.S. 62 (1986), 256-360.

[4] C. O. Ewald, Hochschild Homology and de Rham Cohomology of Stratifolds, Heidelberg, 2002.

[5] J.L. Loday and D. Quillen, Cyclic homology and the Lie algebra homology of matrices, Comment. Math. Helvetici 59 (1984), 565-591.

[6] G. I. Sharygin, Local formulae for characteristic classes of a principal $G L_{n}$-bundle, Sbornik: Mathematics 199 (2008), 1547-1577.

[7] N. Teleman, Microlocalisation de l'homologie de Hochschild, C. R. Acad. Sci. Paris Série I 326 (1998), 1261-1264.

Jan Kubarski, Institute of Mathematics, Lodz University of Technology, Wólczańska 215, 90-924 Łódź, Poland 